$\overline{A E E T}$

ASOCIACIÓN ESPAÑOLA

DE ECOLOGÍA TERRESTRE

\title{
En defensa de una teoría Gaia orgánica
}

\author{
C. de Castro Carranza ${ }^{1, *}$ \\ (1) Dpto. Física Aplicada. Universidad de Valladolid. Campus Miguel Delibes. 47010-Valladolid
}

*Autor de correspondencia: C. de Castro Carranza [ccastro@termo.uva.es]

> Recibido el 15 de noviembre de 2011, aceptado el 27 de mayo de 2013.

\begin{abstract}
De Castro Carranza, C. (2013). En defensa de una teoría Gaia orgánica. Ecosistemas 22(2):113-118. Doi.: 10.7818/ECOS.2013.22-2.17 Las críticas a la hipótesis Gaia de Lovelock (Lovelock y Margulis 1974, Lovelock 1979) convencieron a éste y otros teóricos de la teoría Gaia (Lenton 1998, 2004, Lovelock 2003, Volk 2010), hasta el punto de que se ha rechazado esta hipótesis por todos ellos. Sin embargo, estas críticas parten de presuposiciones reduccionistas y, en gran medida, prejuicios mecanicistas, y son, por tanto, discutibles. En este artículo se defiende el desarrollo de una teoría Gaia científica partiendo de la hipótesis inicial de Lovelock: Gaia es un organismo evolutivo teleológico no necesariamente compatible con la teoría de la selección natural de Darwin (Castro 2008, 2011).
\end{abstract}

Palabras clave: selección natural, superorganismo, paradigma orgánico, teleología

De Castro Carranza, C. (2013). A defence of an organic Gaia theory. Ecosistemas 22(2):113-118. Doi.: 10.7818/ECOS.2013.22-2.17 Lovelock and other theorists of the Gaia Theory (Lenton 1998, 2004, Lovelock 2003, Volk 2010) were convinced by their critics, and modified their initial ideas about Lovelock's Gaia Hypothesis (Lovelock and Margulis 1974, Lovelock 1979). Nevertheless, those criticisms begun with reductionistics presuppositions and, to a great extent, with mechanistic prejudices too, and are, therefore, disputable. In this paper an attempt to develop a scientific Gaia Theory from the initial Lovelock hypothesis is shown: Gaia is a teleological evolving organism, not necessarily based on or even compatible with Darwin natural selection.

Key words: natural selection, superorganism, organic paradigm, teleology

\section{Introducción}

El cambio climático, la pérdida de biodiversidad y de hábitat, el acceso al agua potable y otros problemas ambientales constituyen ejemplos claros de que la humanidad necesita una visión global (holística) de la Tierra (véase por ejemplo "La Carta de la Tierra" promovida por las Naciones Unidas en 2000).

Las teorías derivadas de la hipótesis Gaia de Lovelock y Margulis (Lovelock y Margulis 1974, Lovelock 1979) son quizás las mejores y también las más controvertidas (Kirchner 2003) de las que trabajan con esa perspectiva global, a la vez que abren la posibilidad de profundizar en el paradigma científico y filosófico conocido como organicismo.

La hipótesis inicial de Gaia de Lovelock establece que el conjunto de los seres vivos se comporta como si fuese un superorganismo capaz de regular la composición de la atmósfera y el clima en su beneficio.

Aunque Lovelock, Margulis y otros de sus seguidores como Lenton (Margulis 1998, Lovelock2003, 2004, Margulis y Sagan 2003, Lenton 2004) se han desdicho en varias ocasiones de esta hipótesis, en especial negando que Gaia fuese un organismo, tanto ellos como sus críticos han reconocido que ésta idea ha sido inspiradora (Volk 2004) y se convirtió en fructífera como generadora de otras hipótesis acerca de cómo los procesos biológicos influyen o coevolucionan con los procesos fisico-químicos de la atmósfera, los suelos y los océanos (Kirchner 2003).

Kirchner (1989) hace una clasificación de las distintas hipótesis e interpretaciones que se hacen de Gaia. Esta "taxonomía" se ha venido utilizando desde entonces (e.g. Free and Barton 2007) y la representamos en las Figuras 1 y 2.

\section{Críticas a las hipótesis Gaia fuertes}

Las críticas a la hipótesis inicial de Lovelock vinieron principalmente de la mano de teóricos evolucionistas neodarvinistas, a pesar de que en principio la hipótesis se enmarcaba más en la ciencia ecológica que en la teoría de la evolución de las especies de Darwin.

Para Dawkins (1982) la hipótesis era incompatible con la selección natural de Darwin pues Gaia parecía implicar relaciones cooperativas por parte de organismos egoístas cuando, si ella misma fuese un organismo, debería haber evolucionado a partir de la selección natural y para ello debería haber competido y sido seleccionada con otras gaias o planetas.

Desde el darvinismo parece imposible que la optimización global que supone Gaia provenga de la optimización local de los organismos en su lucha por la existencia (Maynard-Smith 1988). Los organismos se limitan a adaptarse a su entorno aunque en ese proceso lo modifiquen. La hipótesis Gaia fuerte implica que es ella la que adapta el entorno en beneficio del conjunto de los organismos, reduciendo pues la necesidad de adaptación. Gaia tiende de esta forma a dejar con menor poder explicativo a la teoría de la selección natural. Remarquemos por qué son fuerzas opuestas: Si los organismos adaptan el entorno ya no tienen que adaptarse a él, y si se adaptan a él, no tienen que "adaptarlo" para sí (Saunders 1994, Castro 2008, 2011). Es interesante destacar que la discusión actual sobre Gaia se centra solo en la Gaia homeostática (Lenton y Wilkinson 2003, Lovelock 2003, Free y Barton 2007) y a su vez esta discusión está anclada en si aquellas fuerzas son totalmente opuestas e incompatibles (en especial cuando la influencia es de escala planetaria) o si pueden de alguna manera ser complementarias o el resultado de una bidireccionalidad de interacciones. 


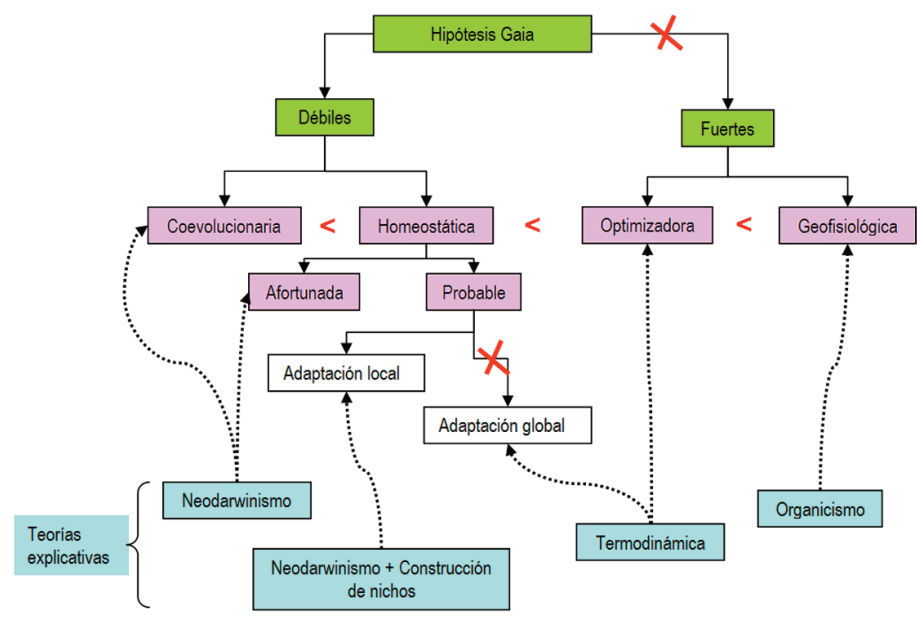

Figura 1. Adaptada de Kirchner (1989) y Free y Barton (2007). Se señalan con una cruz roja las hipótesis que se consideran implausibles o erróneas según estos autores. El símbolo < entre hipótesis, indicaría que las hipótesis más fuertes contendrían a las más débiles. La hipótesis Gaia inicial formulada por Lovelock estaría dentro de las consideradas fuertes.

La hipótesis "coevolucionaria" reconoce que la vida y el entorno se modifican mutuamente (co-evolucionan); no es considerada una hipótesis nueva y sería explicada por la teoría convencional neodarvinista. La hipótesis "homeostática" establece que las condiciones del entorno están restringidas a un rango habitable a través de realimentaciones desde la biota, estas condiciones de habitabilidad ocurririan por suerte (Gaia afortunada) (Watson 2004), como consecuencia quizás de los desechos de los seres vivos (Volk 2004, 2009), o porque los seres vivos en su proceso de adaptación generan realimentaciones sobre el entorno a escala local (por ejemplo a través de la construcción de nichos, Williams y Lenton 2008, Kylafis y Loreau 2008). La hipótesis "optimizadora" establece que la biosfera mantiene el entorno en un estado óptimo para la vida, quizás a partir de la necesidad de aplicar las leyes de la termodinámica a los seres vivos y la biosfera (Kleidon 2002, 2010, Castro 2008, Karnani y Annila 2009). La hipótesis "geofisiológica" identifica la biota y su entorno y lo califica como (super)organismo; esta hipótesis necesitaría desarrollarse desde una teoría diferente al neodarvismo (teoría Gaia orgánica) (Castro 2008, 2011).

Doolittle (1981) criticó la hipótesis Gaia inicial de Lovelock en base a que los organismos inconscientes no podían planificar y prever conscientemente para regular el planeta, como si negociaran cada año el próximo clima y composición atmosférica. Más adelante Kirchner (1989, 1990, 1991, pero sigue insistiendo indirectamente en 2002, 2003) establece que las hipótesis fuertes suponían que Gaia era un ser teleológico, pues tendría capacidad de previsión y planificación consciente. Para él, la hipótesis se convertía así en una metáfora no científica y cualquier interpretación "fuerte" de la hipótesis Gaia implicaba teleología y no era ciencia y las interpretaciones teóricas más "débiles", no teleológicas, no dejaban de ser ciencia ya conocida y convencional.

El éxito de estas críticas fue casi total pues el propio Lovelock rechazó su hipótesis Gaia (Lovelock 2003) y lo que llama desde finales de los años 80 "teoría Gaia" (ver Fig. 2), no tiene ya mucho que ver con su hipótesis inicial (Kirchner 2003). Para Lovelock la teoría Gaia define un sistema homeostático que emerge de la interacción entre la biota y la biosfera y cuyo resultado son estados que la vida tolera y a los que se puede adaptar por selección natural (condiciones de habitabilidad) (Lenton y Wilkinson 2003, Lovelock 2003).

\section{Primera contra-crítica: Daisyworld}

Lovelock (Watson y Lovelock 1983) primero respondió a las críticas de los neodarvinistas elaborando un modelo matemático conocido por Daisyworld que describe un planeta imaginario poblado por dos clases de margaritas, blancas y negras, de tal forma que las blancas tienden a enfriar el planeta al aumentar la cantidad de luz del Daisysol reflejada y las negras lo contrario. El resultado, para ciertos parámetros, es que las poblaciones de margaritas se ajustan y la temperatura queda regulada en un entorno adecuado

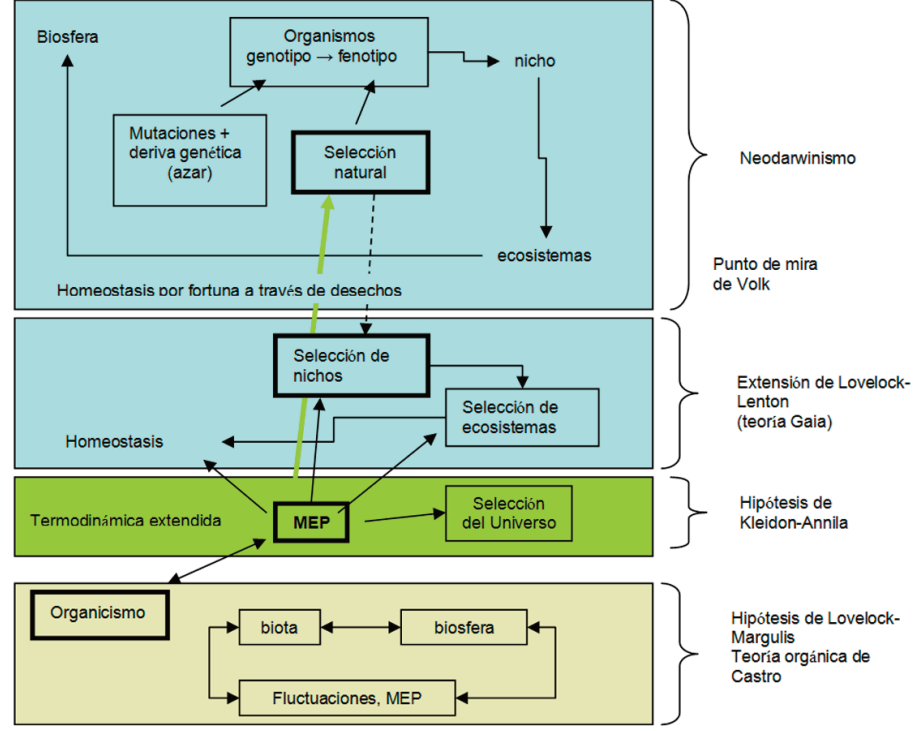

Figura 2. Distintas teorías aplicadas a Gaia. El neodarwinismo admitiría que existen influencias mutuas entre la biosfera y los organismos, pero las influencias están desconectadas en un doble sentido: no admite que los fenotipos determinen los genotipos (sería neolamarckismo), ni admite que el conjunto de organismos determine el entorno hacia estados beneficiosos para el conjunto. Si existe la homeostasis ésta es por fortuna. La idea de Gaia sería un simple cambio de punto de vista que no necesita ninguna hipótesis nueva. Para Lovelock y Lenton, habría que extender el neodarwinismo añadiendo la hipótesis de que además de selección natural de organismos, existe selección en niveles superiores que determinarían la homeostasis y estabilidad que se observa en la biosfera. Añadiría hipótesis propias de la dinámica de sistemas y de los sistemas cibernéticos a las hipótesis neodarwinistas. Kleidon, Annila y otros autores (Kleidon y Lorenz 2005, Karnani y Annila 2009), añaden o sustituyen las hipótesis neodarwinistas por la aplicación de las leyes de la termodinámica y la idea de que en sistemas alejados del equilibrio se producen, a un ritmo máximo, los intercambios energéticos y la entropía, siendo este principio director el que podría explicar la homeostasis de la biosfera e incluso ser el "selector natural" fundamental. La teoría Gaia orgánica, admite las ideas de la termodinámica como principio estabilizador de los sistemas complejos, pero añade, para explicar la evolución y comportamiento de los organismos, hipótesis propias de la biología y de los ecosistemas y no reduce éstas a las leyes de la termodinámica física.

para las margaritas, a pesar de que la luminosidad del sol va aumentando lentamente con el tiempo.

Lovelock argumentó que la regulación de la temperatura se podía considerar una propiedad "gaiana" que surgía de la dinámica de poblaciones y de la interacción de las margaritas con su entorno. Se dice que Daisyworld es compatible con el darvinismo, pues hay una especie de competencia y selección entre margaritas blancas y negras, y la regulación surge sin que el planeta o las margaritas planeen conscientemente cómo conseguirla (Lenton y Lovelock 2000).

En realidad, Daisyworld es tan simple que no tiene sentido hablar de compatibilidad con el darwinismo y de hecho hay quien lo ha interpretado como ejemplo de lo contrario (Saunders 1994, Castro 2008, 2011). Las margaritas no pueden evolucionar, ni cambian, ni se adaptan a la temperatura. No hay verdadera selección natural pues ninguna clase de margarita está mejor adaptada que otra. Sencillamente la mayor parte del tiempo las margaritas conviven, y en todo caso se coordinan con el resultado de regular la temperatura en beneficio mutuo.

Tras el primer Daisyworld se buscan nuevos modelos que introduzcan mecanismos propios de la selección natural darvinista a partir de dinámicas y funciones de los organismos sujetos a ella, de tal forma que se llegue a una regulación de la temperatura (homeostasis). Es decir, que a pesar de que inicialmente Lovelock trata de contestar la idea de que la regulación necesite consciencia por parte de Gaia, los modelos evolucionan luego en el contexto de la teoría Gaia homeostática y no orgánica. Es en esta teoría Gaia ho- 
meostática donde se quiere ver la compatibilidad entre Gaia y la selección natural. De esta forma se ha modificado Daisyworld haciendo que las margaritas puedan cambiar y adaptarse a la temperatura, libremente (Robertson y Robinson 1998) o con restricciones a esa capacidad (Lenton y Lovelock 2000), se ha dejado que entren en el sistema "tramposos", margaritas grises que toman alguna ventaja por mimetizarse con el suelo o por no tener que crear pigmentos blancos o negros, se ha incorporado algún tipo de competencia excluyente (la presencia de una margarita inhibe a la otra), etc. (Lenton y Lovelock 2001, Wood et al. 2008). El resultado es que la regulación se sigue dando aunque de forma menos robusta que en el modelo original cuando se mete una a una cada una de esas características (Lenton y Lovelock 2001); esto tendería a confirmar la idea de Saunders (1994) y Castro (2008) de que la selección natural debilita la capacidad de regulación.

Paradójicamente, a pesar de que modelos de Daisyworld o similares se han ido utilizando con relativa profusión (Wood y Coe 2007, McDonald-Gibson et al 2008, Wood et al. 2008, Boyle et al 2011, Weaver y Dyke 2012), a nadie parece habérsele ocurrido preguntarse qué pasaría si la margarita gris "tramposa" se introduce, no en el modelo original, que no es darvinista, sino en el modelo en el que se simula la capacidad de adaptación a la temperatura con restricciones de Lenton y Lovelock (2000). O añadir a este último modelo competencia excluyente (Lenton y Lovelock 2001), o todo a la vez. El resultado es claro, utilizando los mismos parámetros del modelo original (Watson y Lovelock 1983) y añadiendo las características darwinistas citadas (Lenton y Lovelock 2001), se encuentra que la regulación es destruida (Castro 2008, 2011).

El autor (Castro 2008, 2011) ha diseñado distintos modelos tipo Daisyworld que tratan de eliminar del modelo cualquier parecido al lenguaje propio de la selección natural, al contrario pues de lo que se ha venido haciendo (Wood y Coe 2007, Wood et al. 2008, Boyle et al. 2011). Uno de ellos, el denominado Butterflyworld, imagina un mundo con una sola especie de mariposa con fuerte dimorfismo sexual (macho negro, hembra blanca), para tratar así de romper la idea de competencia y selección natural. Los parámetros del modelo copian el seguido por Lovelock pero en este modelo se cambian las tasas de natalidad y mortalidad de las mariposas respecto a las margaritas y la tasa de transferencia de calor en la superficie del planeta (el parámetro "q" en el Daisyworld original, Watson y Lovelock 1983).

La tasa de crecimiento del área ("natalidad") de las margaritas en Daisyworld es proporcional al área que ocupan $(A)$, al área que falta por ocupar $(x)$ y a un parámetro $B$ que depende de la temperatura local de las margaritas, mientras que la tasa de mortalidad es constante y se elige de un $30 \%$ por generación. De forma que la tasa de variación del área que ocupa un tipo de margarita es:

$$
\frac{d A}{d t}=A \cdot x \cdot B-0.3
$$

En cambio la tasa de crecimiento del área de las mariposas en Butterflyworld depende de la presencia de mariposas del otro sexo y del área que falta por ocupar $(x)$, mientras que es la tasa de mortalidad la que depende del parámetro $B$ y del área que ocupan las mariposas del mismo sexo. De forma que la tasa de variación del área que ocupan los machos es:

$$
\frac{d A_{m}}{d t}=\sqrt{A_{m} \cdot A_{h}} \cdot x-A_{m} \cdot(1-B)
$$

Siendo $A_{m}$ el área ocupada por los machos y $A_{h}$ el área ocupada por las hembras. Así, se maximiza el crecimiento cuando hay tantos machos como hembras y la mortalidad depende de la temperatura (parámetro $B$ ) en proporción al número de machos $\left(A_{m}\right)$.

Aunque en el Butterflyworld original (Castro 2008) se usa un factor de transferencia de calor distinto al de Daisyworld, en la
Figura 3, para que resulte más clara y directa la comparación, representamos el resultado de escoger este factor (q) y el resto de parámetros iguales que en el Daisyworld. Podemos observar que Butterflyworld regula mucho mejor la temperatura y la vida permanece durante más tiempo (ver Fig. 3).

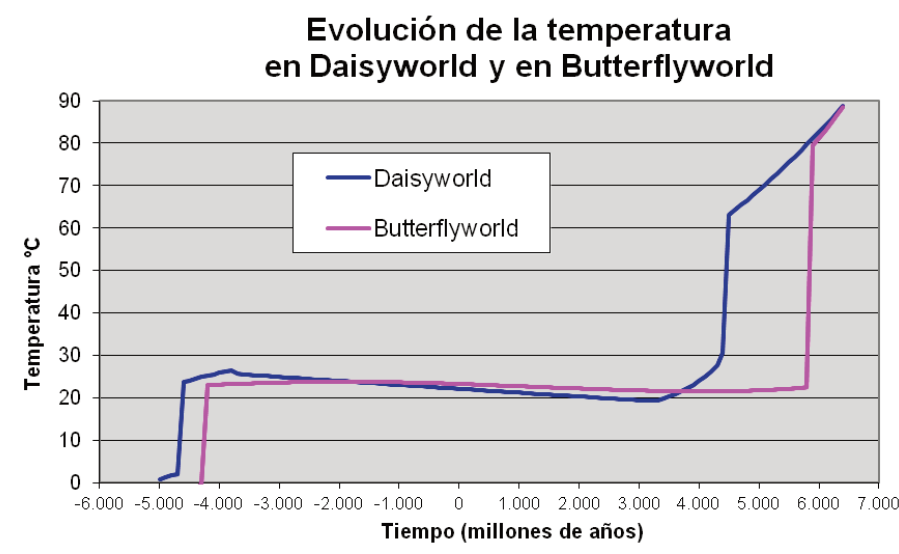

Figura 3. Evolución de la temperatura en Daisyworld y en Butterflyworld cuando la luminosidad de sus soles respectivos va aumentando simulando a una estrella como nuestro Sol. En las zonas de homeostasis (zonas casi horizontales de las gráficas), Daisyworld cambia $7.1^{\circ} \mathrm{C}$ mientras que Butterflyworld lo hace solo en $2.3^{\circ} \mathrm{C}$. El tiempo "cero" corresponde a la misma luminosidad que nuestro Sol tiene hoy.

Aunque la discusión sobre la compatibilidad entre Gaia homeostática y la selección natural sigue abierta (Wood y Coe 2007, Boyle et al. 2011, Weaver y Dyke 2012), y aunque quizás haya que recurrir a otros tipos de "selección", sí que se puede concluir que los Daisyworlds modificados para simular mejor la selección natural y sus efectos sobre la adaptación tienden a perder la regulación o ser menos robustos que aquellos modelos en los que la selección natural es excluida, confirmando la intuición de que la capacidad de adaptación local (por el beneficio del individuo) y la regulación global derivada de la hipótesis Gaia, son fuerzas de sentido opuesto difícilmente compatibles, en este tipo de modelos sencillos, al menos sin añadir nuevas hipótesis (otros tipos de selección).

\section{Otras contra-críticas}

Lovelock (1996) respondió a las críticas iniciales de Doolittle y Dawkins calificándolas de entretenimiento, acusando a estos autores de centrarse sólo en la adaptación de los organismos al cambio ambiental y no en ver la abundante evidencia de las interacciones recursivas entre vida y entorno. Para Lovelock (1996), estas críticas eran dogmáticas y absurdas. Sin embargo, pocos años después, Lovelock rechaza su propia hipótesis inicial y termina reconociendo que esas críticas eran acertadas. Poco a poco va modificando su teoría desde la interpretación geofisiológica (gaia orgánica) hacia una interpretación limitada (gaia homeostática), construyendo una teoría Gaia que "extiende, no contradice, la visión de Darwin, de igual forma como la relatividad amplía, no niega, la física newtoniana" (Lovelock 2003).

Irónicamente, las críticas a la hipótesis de los teóricos evolucionistas son erróneas para algunos autores (Abram 1996, Rawles 1996, Sahtouris 1996), incluso superficiales (Sagan y Whiteside 2004) y quedaron al descubierto relativamente pronto, incluso en publicaciones en las que Lovelock y sus seguidores (Lovelock 1996, 2004, Margulis 1996, Lenton 2004) y algunos de sus críticos (Volk 2004) participaban, quizás porque en su mayor parte vinieron desde la filosofía de la ciencia y no de científicos teóricos de Gaia. Autores como Lovelock, que se han quejado repetidamente del reduccionismo en el método científico y de la excesiva compartimentalización de la ciencia, no han sido aparentemente conscientes de las contundentes contra-argumentaciones a las críticas de su hipótesis Gaia inicial (Lovelock 2004). 
Además, tanto Doolittle como Kirchner y posteriormente otros autores de forma implícita (Free y Barton 2007, Wood et al. 2008, Jax 2010) prejuzgan la imposibilidad de que Gaia sea un organismo ya que se ve al organicismo como una simple metáfora no científica.

Como se ha remarcado por otros autores (Abram 1996, Rawles 1996, Sahtouris 1996), el mecanicismo implícito en la teoría neodarvinista es también una metáfora o si se quiere, una visión o paradigma científico. Es más, "en perspectiva histórica, la filosofía mecanicista de Descartes y Newton emerge como una anomalía en la ciencia natural occidental" (Scofield 2004) y es la filosofía organicista la que tiene una más larga tradición que no ha desaparecido ni mucho menos con el surgimiento y empuje del mecanicismo.

Por tanto, si Gaia fuese un organismo, la ciencia que trabajara con este esquema sería científica y sus hipótesis testables, de forma análoga a como la medicina, la fisiología, la biología y muchas otras ramas científicas estudian organismos. Es falso, como argumentan algunos autores (Kirchner 2003, Free y Barton 2007), pensar que una Gaia orgánica no es testable, porque las propiedades y funciones de ver a Gaia como un organismo son distintas de las que se puede esperar de una Gaia homeostática o simplemente inexistente.

La teoría no solo trataría de explicar la realidad, sino que haría predicciones falsables (y por tanto es testable) (Castro 2008, 2011). Un ejemplo: la teoría predice la "muerte celular programada" en organismos unicelulares, predice que debió surgir evolutivamente primero en las bacterias (Gaia es anterior a los organismos pluricelulares) y que debe estar omnipresente en las distintas clases de organismos. La muerte celular programada en unicelulares en cambio es algo difícil de explicar desde el darvinismo que de hecho hizo la predicción contraria (Vaux et al 1994, Ameisen 1996). De hecho, antes de su descubrimiento en organismos pluricelulares, había indicios experimentales de que la muerte celular programada operaba en unicelulares (Whittingham y Raper 1960, Wireman y Dworkin 1976) pero la "creencia" en la idea darvinista de que ningún organismo en la naturaleza podía cometer suicidio, ya que la selección natural selecciona la vida y no la muerte, impidió identificar correctamente esos indicios porque se presuponía que "obviamente" debía ser un mecanismo exclusivo de los organismos pluricelulares (Ameisen 2002). Más adelante se presupuso, cuando ya se había descubierto en unicelulares, que probablemente había evolucionado primero en pluricelulares y que por transferencia horizontal genética la habían adquirido algunos organismos unicelulares (Vaux et al. 1994). Para finalmente especular que quizás surgió tan pronto como la vida misma (Ameisen 2002). Actualmente se está reconociendo su gran ubicuidad a la vez que se sigue discutiendo la dificultad explicativa desde el neodarwinismo (Duran et al. 2011, Kaczanowski et al 2011).

Dawkins (1982) y posteriormente Volk (2009) en su crítica a Gaia, llegan a definir como organismo a aquel que encaja en la teoría evolutiva que defienden, y excluyen la hipótesis Gaia porque un organismo solo puede surgir de la selección natural, siendo ésta, parte de una teoría científica que trata de explicar un hecho observacional: la evolución de los organismos. Esto implicaría que si el darwinismo fuese falseable y se falseara (Sandín 2006) tendríamos que concluir que Dawkins no es un organismo. No se debería definir un "objeto" de la realidad exclusivamente a partir de una teoría, se debe definir a través de sus propiedades, sin limitar estas a las que se asignen por una teoría particular, que además es contestada aunque sea minoritariamente (Saunders 1994, Ho 1996, Sandin 1997, 2006, 2009, Margulis y Sagan 2003, Santini y Galleni 2004, Castro 2008, 2011)

Este error está implícito en algunos autores que al revisar la teoría Gaia recientemente, descartan las hipótesis Gaia geofisiológicas, considerándolas que son "obviamente" erróneas (Free y Barton 2007) apoyándose en una definición de organismo muy estrecha, incluso dando como propiedad necesaria de organismo la existencia de una genética o herencia basada en el ADN con la capacidad intrínseca de evolucionar a través de la selección natural (Fenchel 2002).
Es decir, pese a las críticas desde la filosofía de la ciencia, la mayoría de los científicos usan presupuestos y prejuicios filosóficos de una metodología científica particular para descartar como imposibilidad científica una teoría Gaia "fuerte", a pesar de que en este tema se opongan a muchos científicos epistemólogos o filósofos científicos. En definitiva, muchos biólogos evolucionistas se oponen, sin saberlo, a los filósofos de la ciencia, no en la interpretación de una teoría científica, sino en la definición de qué es ciencia, campo en el que no son necesariamente expertos frente a los epistemólogos o los filósofos.

Por tanto, parece que la definición de organismo es un tema clave en esta discusión. Lovelock, de hecho, discute las distintas aproximaciones a lo que podría ser una definición de vida o de organismo, reconociendo la dificultad de su definición pero remarcando que la visión neo-darvinista es una de entre varias (Lovelock 2000). Margulis (1998) excluye de la definición de organismo a Gaia con el argumento de una propiedad que tienen los organismos aparentemente secundaria: no viven de sus propios desechos. Otros autores, desde una visión física (termodinámica) se centran en los elementos que tienen en común con sistemas alejados del equilibrio estabilizados por la entrada de energía y la producción de entropía (Kleidon y Lorenz 2005). Fenchel (2002) sí pretende dar una definición de los vivientes a partir de sus propiedades: organización celular, sensibilidad (respuesta a estímulos), metabolismo, desarrollo, reproducción, regulación y homeostasis. Aunque luego, de forma excluyente, añade la herencia (sistema genético basado en $A D N$ ) que permite la adaptación y evolución a través de la selección natural. Gaia en realidad tiene estas propiedades salvo la reproducción (Lovelock 2000) y la adaptación a través de la selección natural; pero ésta última no es una propiedad de los vivientes sino una teoría explicativa de sus propiedades y evolución. Y aunque la reproducción es importante (una hormiga obrera no se reproduce, ni puede en toda su vida), en realidad lo que sí es una propiedad importante es que un organismo ha sido reproducido a partir de otro organismo, y la evolución misma, como hecho observacional de los organismos -otra propiedad-, implica que de forma gradual o a saltos (e.g. simbiogénesis) el organismo que ha sido reproducido lo ha podido ser por otro organismo "anterior": por ejemplo, al aparecer por primera vez un liquen como nueva especie, derivó de dos especies anteriores y no de un "padre" necesariamente parecido o igual al "hijo". En este sentido, Gaia derivaría de los organismos y ecosistemas que la conforman, como una macro-simbiosis (Castro 2008), y en este sentido proviene de una reproducción.

Sin pretender ser excluyente en la definición, organismo sería aquel ser altamente complejo con funciones de autorreparación, metabolismo, ciclado elevado de materia, evolutivo, reproducido y con potencial reproductivo a lo largo de su historia. Entonces, tanto una bacteria, como un árbol, como quizás un termitero y como Gaia, lo serían y se podría comprobar para cualquier otro candidato que se propusiera (Castro 2008, 2011).

De las anteriores características que definen a los organismos emergería una propiedad cualitativamente diferente: el carácter teleológico intrínseco o inherente.

Teleológico es un término inventado y empleado por los filósofos, como Kant, para los cuales significa que tiene o posee propósitos o fines (Sagan y Whiteside 2004, Jax 2010), lo que de hecho se puede aplicar a las máquinas humanas, diseñadas con un propósito asignado externamente por la persona que la fabricó, pero también se aplica a los organismos (Volk 2009), como una ave construyendo un nido, siendo en este caso el propósito no algo exterior al ave sino una emergencia de su ser como organismo, una propiedad inherente al mismo (Castro 2008, Jax 2010).

Tanto Doolittle, como Kirchner y a partir de ellos prácticamente todos los científicos teóricos de Gaia y sus críticos (Kirchner 1989, 2003, Lenton 2004, Lovelock 2004, Wood y Coe 2007, Wood et al. 2008, Weaver y Dike 2012) han empleado y siguen empleando erróneamente el término "teleológico" por excluirlo del ámbito cien- 
tífico e incluso por confundirlo con previsión y planificación consciente, algo que en principio no es aplicable a ningún organismo salvo el ser humano (Moody 2012 sigue remarcando este error recientemente). La huida en la biología del término teleológico llega a la paradoja de inventarse términos nuevos como teleonomía (Monod 1981) o a admitir que los "verdaderos" organismos son "como sí" tuvieran una teleología interna, pero que esta teleología es más un principio heurístico perteneciente más al mundo de las ideas que a un factor real de la naturaleza (Jax 2010, pág. 76); todo ello para no tener que enfrentarse a una realidad fácilmente observable, que los seres vivos hacen las cosas con propósitos propios.

Si bajo el paradigma mecanicista se puede explicar "cómo" hace un nido un ave, es bajo el organicismo con el que los etólogos, biólogos y ecólogos se preguntan lícitamente y con herramientas científicas "para qué" (el propósito) hace un nido un ave. No es solo una cuestión heurística (Jax 2010), pues toda rama científica (incluida la física), es una ciencia del como sí, por lo que si un organismo se comporta como sí tuviera propósitos inherentes y Gaia tiene las propiedades más importantes de lo que denominamos organismo (es como si fuese un super-organismo), entonces, se puede hacer ciencia de la hipótesis de que Gaia es un organismo.

Turner (2000, 2004), por ejemplo, analiza los termiteros de Macrotermes michaelseni como un organismo extendido, de tal forma que cuando estudia la homeostasis del nido de estas termitas dice que sigue siendo una cuestión abierta el "propósito al que sirve la homeostasis del nido", y concluye que puede ser que esta homeostasis refuerce la simbiosis entre las termitas y los hongos que éstas "cultivan". Es el carácter teleológico que se presupone en el superorganismo el que da lugar a la investigación y al descubrimiento de las interacciones con otros organismos, en este caso con un segundo hongo. Estos descubrimientos difícilmente se habrían hecho bajo el paradigma mecanicista.

El origen y evolución de los termiteros pueden tratar de explicarse desde el darvinismo, pero el comportamiento del organismo extendido una vez formado no se describe con esta teoría: no se dice que las termitas del mismo termitero compitan entre ellas por los recursos, ni siquiera que lo hagan con el hongo que cultivan. Vemos el termitero como un todo de partes coordinadas en los que la selección natural no se aplica ni tiene sentido hacerlo.

La teoría Gaia orgánica (Castro 2008, 2011) defiende que existe una integración de sistemas complejos (Sandin 2006) y orgánicos desde la bacteria hasta la misma Gaia, de tal forma que existiría una jerarquía de niveles (holarquía en la terminología de Volk 1995), en la que en cada nivel tendríamos un organismo. Los niveles superiores estarían compuestos por organismos que combinados y coordinados, en general bajo simbiosis, forman un todo mayor -la simbiogénesis de Margulis (1998) formaría parte de esta teoría-. Cuando este todo se va constituyendo en un superorganismo la autonomía y los objetivos que poseían los organismos primitivos se va transfiriendo (Wieser 1997) al nuevo superorganismo. De esta forma, las células eucariotas son el resultado de la combinación y coordinación simbiótica de células procariotas, los organismos pluricelulares son a su vez el resultado de la coordinación de células eucariotas y procariotas unicelulares, los termiteros serían superorganismos resultado de la simbiosis de pluricelulares y unicelulares eucariotas y procariotas, y así hasta llegar a Gaia como organismo resultado de la simbiosis y coordinación de múltiples simbiosis de organismos.

Siempre que encontremos una entidad suficientemente cerrada y estructurada, con propiedades orgánicas, se calificaría de organismo. Así, un termitero o una colmena lo serían mientras que un banco de peces, una plantación de eucaliptos o una ciudad humana, no. En el caso de una colmena observamos las propiedades que pediríamos a un organismo: estructura identificable, reproducción, metabolismo, incluso homeostasis de la temperatura. Y como tal organismo, observaríamos propósitos inherentes de parecida cualidad a los que observamos en un ave construyendo un nido. Así como los órganos de un ave, le sirven al ave (su "telos" o pro- pósitos se dirigen al ave), las termitas o abejas sirven al termitero o la colmena. Entendemos el termitero como un superorganismo precisamente porque sus "organismos", las termitas y sus simbiontes, trabajan (y se sacrifican si es necesario) para los fines no de sí mismos o de otra termita, sino del termitero como conjunto y sus funciones. El termitero como conjunto "selecciona" las funciones y comportamientos de sus termitas y, dentro de él, no se habla de selección natural entre termitas. En el caso de un banco de peces, aunque observamos ciertos comportamientos de conjunto, no definimos el banco en sí como el objetivo de los peces, ni observamos propiedades o funciones metabólicas, ciclado de materiales, etc.; no sería un organismo.

Puede que el darvinismo y el neodarvinismo expliquen para sus proponentes el origen y, también, el comportamiento ecológico de los organismos. Sin embargo, a partir de la escala de los ecosistemas tiene claras dificultades explicativas (Lenton y Wilkinson 2003). Una teoría orgánica de Gaia no tiene pues porqué buscar la compatibilidad con la teoría de Darwin ya que sus ámbitos de aplicación son diferentes.

Análogamente pasa con la teoría gravitatoria de Newton y de Einstein pues cada una se aplica a ámbitos distintos, la primera para casos en que las velocidades y las masas son pequeñas (baja energía) y la segunda al contrario (aunque se pueda aplicar también a bajas energías).

$\mathrm{Ni}$ siquiera se puede decir que la teoría de Einstein es compatible, congruente o una extensión de la teoría de Newton, algo que Lovelock (2003) señala al comparar su teoría con la teoría de la selección natural. Los objetos que manejan las teorías físicas no son siempre los mismos: masas y fuerzas para Newton, distorsiones del espacio-tiempo para Einstein. Y cuando los conceptos se parecen, puede haber contradicciones entre las teorías: un fotón lanzado desde un cohete suma su velocidad a la del cohete para un Newton en reposo relativo, pero para Einstein en cambio no. Son teorías contradictorias e incompatibles en este punto. La teoría de Einstein no es pues una extensión (que añade solo nuevas hipótesis) de la teoría de Newton, sino que utiliza un marco mental y matemático muy diferente al de Newton e hipótesis diferentes que entran en contradicción si las comparamos entre sí

Las teorías Gaia de Lovelock, Lenton y Volk, al alejarse del paradigma orgánico y adaptarse al mecanicismo darvinista han perdido buena parte de la oportunidad de convertirse en los equivalentes de una revolución cuántica o relativista de la ciencia.

\section{Referencias}

Abram, D. 1996. The mechanical and the organic: epistemological consequences of the Gaia hypothesis. En: Bunyard, P. (ed.), Gaia in Action, pp. 234-247. Floris Books. Edinburgh. UK.

Ameisen, J.C. 1996. The Origin of programmed cell death. Science 272:1278-1279

Ameisen, J.C. 2002. On the origin, evolution, and nature of programmed cell death: a timeline of four billions years. Cell Death and Differentiation 9:367-393

Boyle, R.A., Lenton T.M., Watson, A.J. 2011. Symbiotic physiology promotes homeostasis in Daisyworld. Journal of Theoretical Biology 274:170-182

Castro, C. 2008. El Origen de Gaia: una teoría holista de la evolución. Editorial Abecedario. Badajoz. España.

Castro, C. 2011. Teoría Gaia Orgánica. Editorial Bubok. Disponible en: http://www.bubok.es/libros/199109/Teoria-Gaia-Organica

Dawkins, R. 1982. The Extended Phenotype. Freeman. Oxford. UK.

Doolittle, W.F. 1981. Is Nature really motherly? CoEvolution Quaterly 29:5863

Duran, P.M., Rashidi, A., Michod, P.E. 2011. How an organism dies affects the fitness of its neighbords. The American naturalist 177(2):224-232

Fenchel, T. 2002. The origin and early history of life. Oxford University Press.

Free, A., Barton, N.H. 2007. Do evolution and ecology need the Gaia hypothesis? Trends in Ecology and Evolution 22(11):611-619

Ho, M.W. 1996. Natural Being and Coherent Society. En: Bunyard, P. (ed.), Gaia in action, pp. 286-307. Floris Books. Edinburgh. UK. 
Jax, K. 2010. Ecosystem Functioning. Cambridge University Press. UK.

Kaczanowski, S., Sajid, M., Reece, S.E. 2011. Evolution of apoptosis-like programmed cell death in unicellular protozoan parasites. Parasites and Vectors 4(44):1-8

Karnani, M., Annila, A. 2009: Gaia again. Biosystems 95:82-87

Kirchner, J.W. 1989. The Gaia hypothesis: Can it Be Tested?. Reviews of Geophysics 27:223-235

Kirchner, J.W. 1990. Gaia. Metaphor unfalsifiable. Nature 345:470

Kirchner, J.W. 1991. The Gaia hypotheses: are they testable? Are they useful? En: Schneider , S.H., Boston, P.J. (eds.), Scientists on Gaia pp. 3846. MIT Press. Cambridge. UK.

Kirchner, J.W. 2002. The Gaia Hypothesis: Fact, Theory and Wishful Thinking" Climatic Change 52:391-408

Kirchner, J.W. 2003. The Gaia hypothesis: Conjectures and Refutations. Climatic Change 58:21-45

Kleidon, A. 2002. Testing the effect of life on Earth's functioning: How gaian is the Earth system? Climatic Change 52:383-389

Kleidon, A. 2010. Life, hierarchy and the thermodynamic machinery of planet Earth. Physics of Life reviews 7:426-460

Kleidon, A., Lorenz, R.D. (eds) 2005. Non-equilibrium thermodynamcis and the production of entropy. Life, Earth and beyond.Springer-Verlag, Heidelberg, Germany

Kylafis, G., Loreau, M. 2008. Ecological and evolutionary consequences of niche construction for its agent. Ecology Letters 11(10):1072-1081

Lenton, T.M. 1998. Gaia and natural selection. Nature 394:439-447

Lenton, T.M. 2004. Clarifying Gaia: Regulation with or without Natural Selection. En: Schneider, S.H., et al. (eds.), Scientists Debate Gaia, pp 15-25. MIT Press. Cambridge, MA. USA

Lenton, T.M., Lovelock, J.E. 2000. Daisyworld is Darwinian: constraints on adaptation are important for planetary sel-regulation. Journal of Theoretical Biology 206:109-114

Lenton, T.M., Lovelock, J.E. 2001. Daisyworld revisited: quantifying biolog ical effects on planetary self-regulation. Tellus 53B:288-305

Lenton, T.M., Wilkinson, D.M. 2003. Developing the Gaia Theory. Climatic Change 58:1-12.

Lovelock, J.E. 1979. Gaia: a New Look at Life on Earth. Oxford University Press. UK

Lovelock, J.E. 1996. The Gaia Hypothesis. En: Bunyard, P. (ed.), Gaia in Action. pp 15-33 Floris Books. Edinburgh. UK.

Lovelock, J.E. 2000. Gaia. The practical science of planetary medicine. Oxford University Press. UK.

Lovelock, J.E. 2003. The living Earth. Nature 426:769-770

Lovelock, J.E. 2004. Reflections on Gaia. En: Schneider, S.H., et al. (eds.), Scientists Debate Gaia. pp. 1-5. MIT Press. Cambridge, MA. USA.

Lovelock, J.E., Margulis, L. 1974. Atmospheric Homeostasis by and for the Biosphere: The Gaia hypothesis. Tellus 26:2-10

McDonald-Gibson, D., Dyke, J.G., DiPaolo, E.A., Harvey, I.R. 2008. Environmental regulation can arise under minimal assumptions. Journal of Theoretical Biology 251:653-666

Margulis, L. 1996. James Lovelock's Gaia. En: Bunyard, P. (ed.). Gaia in Action. pp. 54-64. Floris Books. Edinburgh. UK.

Margulis, L. 1998. The Symbiotic Planet: a new look at evolution. Phoenix. London. UK.

Margulis, L., Sagan, D. 2003. Captando Genomas: una teoría sobre el origen de las especies. Kairós. Barcelona. España.

Maynard-Smith, J. 1988. Evolutionary progress and levels of selection. En: Nitecki, M. (ed.). Evolutionary Progress, pp. 219-230. University of Chicago Press. Chicago, IL. USA.

Moody, D.E. 2012. Seven misconceptions regarding the Gaia hypothesis Climatic Change 113:277-284.

Monod, J. 1981. El azar y la necesidad. Metatemas. Tusquets editores. Barcelona, España.
Rawles, K. 1996. Ethical implications of the Gaia thesis. En: Bunyard, P. (ed.). Gaia in Action pp. 308-323. Floris Books. Edinburgh. UK.

Robertson, D., Robinson, J. 1998. Darwinian Daisyworld. Journal of Theoretical Biology 195:129-134

Sagan, D., Schneider, E. 2008. La termodinámica de la vida. Tusquets. Barcelona, España.

Sagan, D., Whiteside, J.H. 2004. Gradient Reduction Theory: thermodynamics and the Purpose of Life. En: Schneider, S.H., et al. (eds.), Scientist Debate Gaia. pp 173-186. MIT Press. Cambridge, MA. USA.

Sahtouris, E. 1996. The Gaia Controversy: a Case for the Earth as an Evolving Organism. En: Bunyard (ed.) Gaia in Action. pp. 324-338. Floris Books, Edinburgh. UK.

Sandín, M. 1997. Teoría sintética: crisis y revolución. Arbor 623-624: 269304

Sandín, M. 2006. Pensando la evolución, pensando la vida. Ediciones Crimentales. Murcia.

Sandín, M. 2009. En busca de la biología: reflexiones sobre la evolución. Asclepio 61(2):141-176

Santini, F., Galleni, L. 2004. Stability and Instability in Ecological Systems: Gaia Theory and Evolutionary Biology. En: Schneider, S.H., et al (eds.), Scientists Debate Gaia. pp 353-362. MIT Press. Cambridge, MA. USA.

Saunders, P.T. 1994. Evolution without Natural Selection: Further Implications of the Daisyworld. Tellus 35B:284-289

Scofield, B. 2004. Gaia: the living Earth. 2500 years of precedents in natura science and philosophy. En: Schneider, S.H., et al. (eds.). Scientists Debate Gaia. Pp. 151-160. MIT Press. Cambridge, MA. USA.

Turner, J.S. 2000. The extended organism: the physiology of animal-built structures. Harvard University Press. Cambridge. UK.

Turner, J.S. 2004. Gaia, extended organisms and emergent homeostasis. En: Schneider, S.H., et al. (eds.) Scientists Debate Gaia. pp 57-70. MIT Press. Cambridge, MA. USA.

Vaux, D.L., Haecker, G., Strasser, A. 1994. An evolutionary perspective on apoptosis. Cell 76:777-779

Volk, T. 1995. Mettapaterns. Across space, time and mind. Columbia University Press. New York. USA.

Volk, T. 2004. Gaia is Life in a Wasteworld of By-products. En: Schneider, S.H., et al. (eds.) Scientists Debate Gaia, pp. 27-36 MIT Press. Cambridge, MA. USA.

Volk, T. 2009. How the Biosphere Works. En: Crist, E.,Rinker, B. (eds.) Gaia in Turmoil: Climate Change, Biodepletion, and Earth Ethics in an Age of Crisis, pp. 27-40. MIT Press. Cambridge, MA. USA.

Watson, A.J. 2004. Gaia and Observer Self-selection. En: Schneider, S.H., et al. (eds.) Scientists Debate Gaia. pp. 201-208. MIT Press. Cambridge, MA. USA.

Watson, A.J., Lovelock, J.E. 1983. Biological homeostasis of the global environment : the parable of Daisyworld. Tellus 35B:284-289

Weaver, I.S., Dyke, J.G. 2012. The importance of timescales for the emergence of environmental self-regulation. Journal of Theoretical Biology 313:172-180

Wieser, W. 1997. A major transition in Darwinism. Tree 12(9):367-370

Whittingham, K.B., Raper, W.F. 1960. Non-viability of stalk cells in Dictyostelium PNAS. 46:642-649

Williams, H.T.P., Lenton, T.M. 2008. Environmental regulation in a network of simulated ecosystems. PNAS 105:10432-10437

Wireman, J.W., Dworkin, M. 1976. Developmentally induced autolysis during fruiting body formation by Mixococcus xanthus. Journal of bacteriology 129:796-802

Wood, A.J., Ackland, G.J., Dyke, J.G., Williams, H.T.P., Lenton, T.M. 2008. Daisyworld: a review. Reviews of Geophysics 46:RG1001.

Wood, A.J., Coe, J.B. 2007. A fitness based analysis of Daisyworld. Journal of Theoretical Biology 249:190-197. 